\title{
UNIQUENESS OF SOLUTIONS FOR AN ELLIPTIC EQUATION MODELING MEMS*
}

\author{
PIERPAOLO ESPOSITO $^{\dagger}$ AND NASSIF GHOUSSOUB
}

\begin{abstract}
We show among other things, that for small voltage, the stable solution of the basic nonlinear eigenvalue problem modelling a simple electrostatic MEMS is actually the unique solution, provided the domain is star-shaped and the dimension is larger or equal than 3 . In two dimensions, we need the domain to be either strictly convex or symmetric. The case of a power permittivity profile is also considered. Our results, which use an approach developed by Schaaf [13], extend and simplify recent results by Guo and Wei [7], [8].
\end{abstract}

Key words. MEMS, Stable solutions, Quenching branch.

AMS subject classifications. 35J60, 35B32, 35D10, 35J20

1. Introduction. We study the effect of the parameter $\lambda$, the dimension $N$, the profile $f$ and the geometry of the domain $\Omega \subset \mathbb{R}^{N}$, on the question of uniqueness of the solutions to the following elliptic boundary value problem with a singular nonlinearity:

$$
\left\{\begin{array}{ll}
-\Delta u=\frac{\lambda f(x)}{(1-u)^{2}} & \text { in } \Omega \\
0<u<1 & \text { in } \Omega \\
u=0 & \text { on } \partial \Omega .
\end{array}(S)_{\lambda, f}\right.
$$

This equation has been proposed as a model for a simple electrostatic MicroElectromechanical System (MEMS) device consisting of a thin dielectric elastic membrane with boundary supported at 0 below a rigid ground plate located at height $z=1$. See $[10,11]$. A voltage - directly proportional to the parameter $\lambda$-is applied, and the membrane deflects towards the ground plate and a snap-through may occur when it exceeds a certain critical value $\lambda^{*}$, the pull-in voltage.

In [9] a fine ODE analysis of the radially symmetric case with a constant profile $f \equiv 1$ on a ball $B$, yields the following bifurcation diagram that describes the $L^{\infty}$ norm of the solutions $u$ - which in this case necessarily coincides with $u(0)$ - in terms of the corresponding voltage $\lambda$.

The question whether the diagram above describes realistically the set of all solutions in more general domains and for non-constant profiles, and whether rigorous mathematical proofs can be given for such a description, has been the subject of many recent investigations. See $[3,4,5,7,8]$.

We summarize in the following two theorems some of the established results concerning Figure 1. First, for every solution $u$ of $(S)_{\lambda, f}$, we consider the linearized operator

$$
L_{u, \lambda}=-\Delta-\frac{2 \lambda f}{(1-u)^{3}}
$$

\footnotetext{
*Received August 10, 2008; accepted for publication October 21, 2008.

${ }^{\dagger}$ Dipartimento di Matematica, Università degli Studi "Roma Tre", 00146 Rome, Italy (esposito@mat.uniroma3.it). Research supported by M.U.R.S.T., project "Variational methods and nonlinear differential equations".

${ }^{\ddagger}$ Department of Mathematics, University of British Columbia, Vancouver, B.C. Canada V6T 1Z2 (nassif@math.ubc.ca). Research partially supported by the Natural Science and Engineering Research Council of Canada.
} 


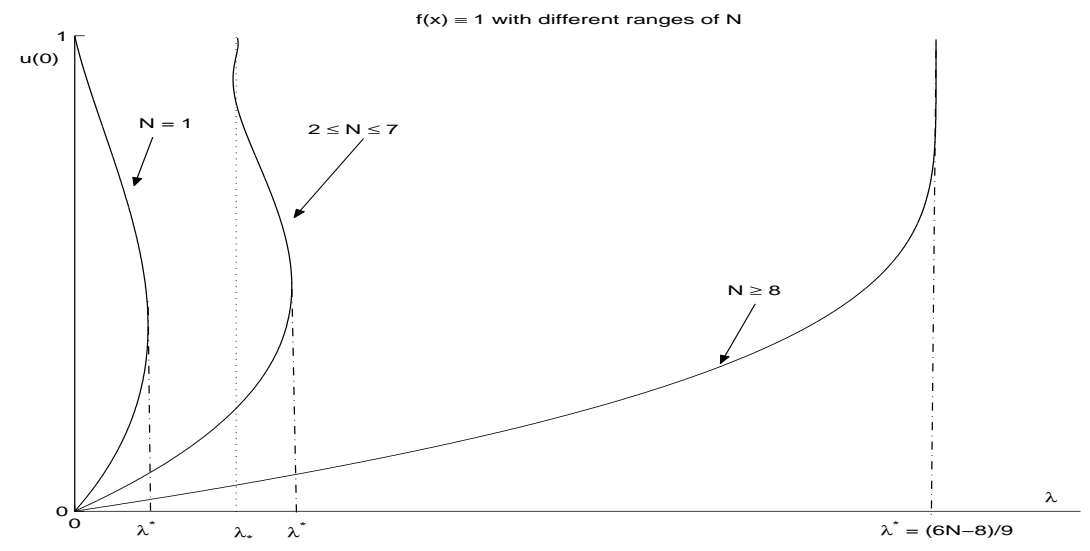

FIG. 1. Plots of $u(0)$ versus $\lambda$ for profile $f(x) \equiv 1$ defined in the unit ball $B_{1}(0) \subset \mathbb{R}^{N}$ with different ranges of $N$. In the case $N \geq 8$, we have $\lambda^{*}=2(3 N-4) / 9$.

and its eigenvalues $\left\{\mu_{k, \lambda}(u) ; k=1,2, \ldots\right\}$ (with the convention that eigenvalues are repeated according to their multiplicities). The Morse index $m(u, \lambda)$ of a solution $u$ is the largest $k$ for which $\mu_{k, \lambda}(u)$ is negative. A solution $u$ of $(S)_{\lambda, f}$ is said to be stable (resp., semi-stable) if $\mu_{1, \lambda}(u)>0$ (resp., $\mu_{1, \lambda}(u) \geq 0$ ).

A description of the first stable branch and of the higher unstable ones is given in the following.

Theorem A $[3,4,5]$. Suppose $f$ is a smooth nonnegative function in $\Omega$. Then, there exists a finite $\lambda^{*}>0$ such that

1. If $0 \leq \lambda<\lambda^{*}$, there exists a (unique) minimal solution $u_{\lambda}$ of $(S)_{\lambda, f}$ such that $\mu_{1, \lambda}\left(u_{\lambda}\right)>0$. It is also unique in the class of all semi-stable solutions.

2. If $\lambda>\lambda^{*}$, there is no solution for $(S)_{\lambda, f}$.

3. If $1 \leq N \leq 7$, then $u^{*}=\lim _{\lambda \uparrow \lambda^{*}} u_{\lambda}$ is a solution of $(S)_{\lambda^{*}, f}$ such that $\mu_{1, \lambda^{*}}\left(u^{*}\right)=$ 0 , and $u^{*}$ - referred to as the extremal solution of problem $(S)_{\lambda, f}$ - is the unique solution.

4. If $1 \leq N \leq 7$, there exists $\lambda_{2}^{*}$ with $0<\lambda_{2}^{*}<\lambda^{*}$ such that for any $\lambda \in\left(\lambda_{2}^{*}, \lambda^{*}\right)$, problem $(S)_{\lambda, f}$ has a second solution $U_{\lambda}$ with $\mu_{1, \lambda}\left(U_{\lambda}\right)<0$ and $\mu_{2, \lambda}\left(U_{\lambda}\right)>0$. Moreover, at $\lambda=\lambda_{2}^{*}$ there exists a second solution $U^{*}:=\lim _{\lambda \downarrow \lambda_{2}^{*}} U_{\lambda}$ with

$$
\mu_{1, \lambda_{2}^{*}}\left(U^{*}\right)<0 \quad \text { and } \quad \mu_{2, \lambda_{2}^{*}}\left(U^{*}\right)=0 .
$$

5. Given a more specific potential $f$ in the form

$$
f(x)=\left(\prod_{i=1}^{k}\left|x-p_{i}\right|^{\alpha_{i}}\right) h(x), \quad \inf _{\Omega} h>0,
$$

with points $p_{i} \in \Omega, \alpha_{i} \geq 0$, and given $u_{n}$ a solution of $(S)_{\lambda_{n}, f}$, we have the equivalence

$$
\left\|u_{n}\right\|_{\infty} \rightarrow 1 \quad \Longleftrightarrow \quad m\left(u_{n}, \lambda_{n}\right) \rightarrow+\infty
$$

as $n \rightarrow+\infty$. 
It was also shown in [4] that the permittivity profile $f$ can dramatically change the bifurcation diagram, and totally alter the critical dimensions for compactness. Indeed, the following theorem summarizes the result related to the effect of power law profiles.

TheOrem B [4]. Assume $\Omega$ is the unit ball $B$ and $f$ in the form

$$
f(x)=|x|^{\alpha} h(|x|), \quad \inf _{B} h>0 .
$$

Then we have

1. If $N \geq 8$ and $\alpha>\alpha_{N}:=\frac{3 N-14-4 \sqrt{6}}{4+2 \sqrt{6}}$, the extremal solution $u^{*}$ is again $a$ classical solution of $(S)_{\lambda^{*}, f}$ such that $\mu_{1, \lambda^{*}}\left(u^{*}\right)=0$.

2. If $N \geq 8$ and $\alpha>\alpha_{N}:=\frac{3 N-14-4 \sqrt{6}}{4+2 \sqrt{6}}$, the conclusion of Theorem A-(4) still holds true.

3. On the other hand, if either $2 \leq N \leq 7$ or $N \geq 8,0 \leq \alpha \leq \alpha_{N}=\frac{3 N-14-4 \sqrt{6}}{4+2 \sqrt{6}}$, for $f(x)=|x|^{\alpha}$ necessarily we have that

$$
u^{*}(x)=1-|x|^{\frac{2+\alpha}{3}}, \quad \lambda^{*}=\frac{(2+\alpha)(3 N+\alpha-4)}{9} .
$$

The bifurcation diagram suggests the following conjectures:

1. For $2 \leq N \leq 7$ there exists a curve $(\lambda(t), u(t))_{t \geq 0}$ in the solution set

$$
\mathcal{V}=\left\{(\lambda, u) \in(0,+\infty) \times C^{1}(\bar{\Omega}): u \text { is a solution of }(S)_{\lambda, f}\right\}
$$

starting from $(0,0)$ at $t=0$ and going to "infinity": $\|u(t)\|_{\infty} \rightarrow 1$ as $t \rightarrow+\infty$, with infinitely many bifurcation or turning points in $\mathcal{V}$.

2. In dimension $N \geq 2$ and for any profile $f$, there exists a unique solution for small voltages $\lambda$.

3. For $2 \leq N \leq 7$ there exist exactly two solutions for $\lambda$ in a small left neighborhhod of $\lambda^{*}$.

Conjectures 1 and 2 have been established for power law profiles in the radially symmetric case [7], and for the case where $f \equiv 1$ and $\Omega$ is a suitably symmetric domain in $\mathbb{R}^{2}[8]$. Indeed, in these cases Guo and Wei first show that

$$
\lambda_{*}=\inf \left\{\lambda>0:(S)_{\lambda, f} \text { has a non-minimal solution }\right\}>0,
$$

and then apply the fine bifurcation theory developed by Buffoni, Dancer and Toland [1] to verify the validity of Conjecture 1 in that case. The fact that $\lambda_{*}>0$ then allows them to carry out some limiting argument and to prove that the Morse index of $u(t)$ blows up as $t \rightarrow+\infty$, which is crucial for showing that infinitely many bifurcation or turning points occur along the curve. Thanks to Theorem A-(5), we shall be able in Section 2 to show the validity of Conjecture 1 in general domains $\Omega$, by circumventing the need to prove that $\lambda_{*}>0$. On the other hand, we shall prove in Section 3 that indeed $\lambda_{*}>0$ for a large class of domains, and therefore we have uniqueness for small voltage. Our proofs simplify considerably those of Guo and Wei [7, 8], and extend them to general star-shaped domains $\Omega$ and power law profiles $f(x)=|x|^{\alpha}, \alpha \geq 0$.

Conjecture 3 has been shown in [3] in the class of solutions $u$ with $m(u, \lambda) \leq k$, for every given $k \in \mathbb{N}$, and is still open in general. 
2. A quenching branch of solutions. The first global result on the set of solutions in general domains was proved by the first author in [3]. By using a degree argument (repeated below), he showed the following result.

TheOREm 2.1. Assume $2 \leq N \leq 7$ and $f$ be as in (1). There exist a sequence $\left\{\lambda_{n}\right\}_{n \in \mathbb{N}}$ and associated solution $u_{n}$ of $(S)_{\lambda_{n}, f}$ so that

$$
m\left(u_{n}, \lambda_{n}\right) \rightarrow+\infty \quad \text { as } n \rightarrow+\infty .
$$

We now introduce some notation from Section 2.1 of [1]. Set

$$
X=Y=\left\{u \in C^{1}(\bar{\Omega}): u=0 \text { on } \partial \Omega\right\}, \quad U=(0,+\infty) \times\left\{u \in X:\|u\|_{\infty}<1\right\},
$$

and define the real analytic function $F: \mathbb{R} \times U \rightarrow Y$ as $F(\lambda, u)=u-\lambda K(u)$, where $K(u)=-\Delta^{-1}\left(f(x)(1-u)^{-2}\right)$ is a compact operator on every closed subset in $\left\{u \in X:\|u\|_{\infty}<1\right\}$ and $\Delta^{-1}$ is the Laplacian resolvent with homogeneous Dirichlet boundary condition. The solution set $\mathcal{V}$ given in (2) rewrites as

$$
\mathcal{V}=\{(\lambda, u) \in U: F(\lambda, u)=0\},
$$

and the projection of $\mathcal{V}$ onto $X$ is defined as

$$
\Pi_{X} \mathcal{V}=\{u \in X: \exists \lambda \text { so that }(\lambda, u) \in \mathcal{V}\}
$$

Proof. In view of Theorem A-(5), we have the equivalence

$$
\sup _{(\lambda, u) \in \mathcal{V}} \max _{\Omega} u=1 \quad \Longleftrightarrow \quad \sup _{(\lambda, u) \in \mathcal{V}} m(u, \lambda)=+\infty .
$$

Arguing by contradiction, we can assume that

$$
\sup _{(\lambda, u) \in \mathcal{V}} \max _{\Omega} u \leq 1-2 \delta, \quad \sup _{(\lambda, u) \in \mathcal{V}} m(u, \lambda)<+\infty
$$

for some $\delta \in\left(0, \frac{1}{2}\right)$. By Theorem 1.3 in [3] one can find $\lambda_{1}, \lambda_{2} \in\left(0, \lambda^{*}\right), \lambda_{1}<\lambda_{2}$, so that $(S)_{\lambda, f}$ possesses

- for $\lambda_{1}$, only the (non degenerate) minimal solution $u_{\lambda_{1}}$ which satisfies $m\left(u_{\lambda_{1}}, \lambda_{1}\right)=0$

- for $\lambda_{2}$, only the two (non degenerate) solutions $u_{\lambda_{2}}, U_{\lambda_{2}}$ satisfying $m\left(u_{\lambda_{2}}, \lambda_{2}\right)=0$ and $m\left(U_{\lambda_{2}}, \lambda_{2}\right)=1$, respectively.

Consider a $\delta$-neighborhood $\mathcal{V}_{\delta}$ of $\Pi_{X} \mathcal{V}$ :

$$
\mathcal{V}_{\delta}:=\left\{u \in X: \operatorname{dist}_{X}\left(u, \Pi_{X} \mathcal{V}\right) \leq \delta\right\} .
$$

Note that (3) gives that $\mathcal{V}$ is contained in a closed subset of $\left\{u \in X:\|u\|_{\infty}<1\right\}$ :

$$
\mathcal{V}_{\delta} \subset\left\{u \in X:\|u\|_{\infty} \leq 1-\delta\right\} .
$$

We can now define the Leray-Schauder degree $d_{\lambda}$ of $F(\lambda, \cdot)$ on $\mathcal{V}_{\delta}$ with respect to zero, since by definition of $\Pi_{X} \mathcal{V}$ (the set of all solutions) $\partial \mathcal{V}_{\delta}$ does not contain any solution of $(S)_{\lambda, f}$ for any value of $\lambda$. Since $d_{\lambda}$ is well defined for any $\lambda \in\left[0, \lambda^{*}\right]$, by homotopy $d_{\lambda_{1}}=d_{\lambda_{2}}$. To get a contradiction, let us now compute $d_{\lambda_{1}}$ and $d_{\lambda_{2}}$. Since the only 
zero of $F\left(\lambda_{1}, \cdot\right)$ in $\mathcal{V}_{\delta}$ is $u_{\lambda_{1}}$ with Morse index zero, we have $d_{\lambda_{1}}=1$. Since $F\left(\lambda_{2}, \cdot\right)$ has in $\mathcal{V}_{\delta}$ exactly two zeroes $u_{\lambda_{2}}$ and $U_{\lambda_{2}}$ with Morse index zero and one, respectively, we have $d_{\lambda_{2}}=1-1=0$. This contradicts $d_{\lambda_{1}}=d_{\lambda_{2}}$, and the proof is complete.

We can now combine Theorem A-(5) with the fine bifurcation theory in [1] to establish a more precise multiplicity result. See also [2].

Observe that $\mathcal{A}_{0}:=\left\{\left(\lambda, u_{\lambda}\right): \lambda \in\left(0, \lambda^{*}\right)\right\}$ is a maximal arc-connected subset of

$$
S:=\left\{(\lambda, u) \in U: F(\lambda, u)=0 \text { and } \partial_{u} F(\lambda, u): X \rightarrow Y \text { is invertible }\right\}
$$

with $\mathcal{A}_{0} \subset S$. Assume that the extremal solution $u^{*}$ is a classical solution so to have $u^{*} \in(\bar{S} \cap U) \backslash S$. Assumption (C1) of Section 2.1 in [1] does hold in our case. As far as condition $(\mathrm{C} 2)$ :

$$
\{(\lambda, u) \in U: F(\lambda, u)=0\} \text { is open in }\{(\lambda, u) \in \mathbb{R} \times X: F(\lambda, u)=0\},
$$

let us stress that it is a weaker statement than requiring $U$ to be an open subset in $\mathbb{R} \times X$. In our case, the map $F(\lambda, u)$ is defined only in $U$ (and not in the whole $X$ ), and then condition (C2) does not make sense. However, we can replace it with the new condition $(\mathrm{C} 2)$ :

$U$ is an open set in $\mathbb{R} \times X$,

which does hold in our context. Since (C2) is used only in Theorem 2.3-(iii) in [1] to show that $S$ is open in $\bar{S}$, our new condtion (C2) does not cause any trouble in the arguments of [1].

Since $\partial_{u} F(\lambda, u)$ is a Fredholm operator of index 0, by a Lyapunov-Schmidt reduction we have that assumptions (C3)-(C5) do hold in our case (let us stress that these conditions are local and $U$ is an open set in $\mathbb{R} \times X)$.

Setting $\bar{\lambda}=0$ and defining the map $\nu: U \rightarrow[0,+\infty)$ as $\nu(\lambda, u)=\frac{1}{1-\|u\|_{\infty}}$, conditions (C6)-(C8) do hold in view of the property $\lambda \in\left[0, \lambda^{*}\right]$. Theorem 2.4 in [1] then applies and gives the following.

TheOrem 2.2. Assume $u^{*}$ a classical solution of $(S)_{\lambda^{*}, f}$. Then there exists an analytic curve $(\hat{\lambda}(t), \hat{u}(t))_{t \geq 0}$ in $\mathcal{V}$ starting from $(0,0)$ and so that $\|\hat{u}(t)\|_{\infty} \rightarrow 1$ as $t \rightarrow+\infty$. Moreover, $\hat{u}(t)$ is a non-degenerate solution of $(S)_{\hat{\lambda}(t), f}$ except at isolated points.

By the Implicit Function Theorem, the curve $(\hat{\lambda}(t), \hat{u}(t))$ can only have isolated intersections. If we now use the usual trick of finding a minimal continuum in $\{(\hat{\lambda}(t), \hat{u}(t)): t \geq 0\}$ joining $(0,0)$ to "infinity", we obtain a continuous curve $(\lambda(t), u(t))$ in $\mathcal{V}$ with no self-intersections which is only piecewise analytic. Clearly, $\partial_{u} F(\lambda, u): X \rightarrow Y$ is still invertible along the curve except at isolated points.

Let now $2 \leq N \leq 7$ and $f$ be as in (1). By the equivalence in Theorem A-(5) we get that $m(\lambda(t), u(t)) \rightarrow+\infty$ as $t \rightarrow+\infty$, and then $\mu_{k, \lambda(t)}(u(t))<0$ for $t$ large, for every $k \geq 1$. Since $\mu_{k, \lambda(0)}(u(0))=\mu_{k, 0}(0)>0$ and $u(t)$ is a non-degenerate solution of $(S)_{\lambda(t), f}$ except at isolated points, we find $t_{k}>0$ so that $\mu_{k, \lambda(t)}(u(t))$ changes from positive to negative sign across $t_{k}$. Since $\mu_{k+1, \lambda(t)}(u(t)) \geq \mu_{k, \lambda(t)}(u(t))$, we can choose $t_{k}$ to be non-increasing in $k$ and to have $t_{k} \rightarrow+\infty$ as $k \rightarrow+\infty$.

To study secondary bifurcations, we will use the gradient structure in the problem. 
Setting $\left(\lambda_{k}, u_{k}\right):=\left(\lambda\left(t_{k}\right), u\left(t_{k}\right)\right)$, we have that $\left(\lambda_{k}, u_{k}\right) \notin S$. Choose $\delta>0$ small so that $\left\|u_{k}\right\|_{\infty}<1-\delta$, and replace the nonlinearity $(1-u)^{-2}$ with a regularized one:

$$
f_{\delta}(u)= \begin{cases}(1-u)^{-2} & \text { if } u \leq 1-\delta, \\ \delta^{-2} & \text { if } u \geq 1-\delta,\end{cases}
$$

and the map $F(\lambda, u)$ with the corresponding one $F_{\delta}(\lambda, u)$. We replace $X$ and $Y$ with $H^{2}(\Omega) \cap H_{0}^{1}(\Omega)$ and $L^{2}(\Omega)$, respectively. The map $F_{\delta}(\lambda, u)$ can be considered as a map from $\mathbb{R} \times X \rightarrow Y$ with a gradient structure:

$$
\partial_{u} \mathcal{J}_{\delta}(\lambda, u)[\varphi]=\left\langle F_{\delta}(\lambda, u), \varphi\right\rangle_{L^{2}(\Omega)}
$$

for every $\lambda \in \mathbb{R}$ and $u, \varphi \in X$, where $\mathcal{J}_{\delta}: \mathbb{R} \times X \rightarrow \mathbb{R}$ is the functional given by

$$
\mathcal{J}_{\delta}(\lambda, u)=\frac{1}{2} \int_{\Omega}|\nabla u|^{2} d x-\lambda \int_{\Omega} f(x) G_{\delta}(u) d x, \quad G_{\delta}(u)=\int_{0}^{u} f_{\delta}(s) d s .
$$

Assumptions (G1)-(G2) in Section 2.2 of [1] do hold. We have that $(\lambda(t), u(t)) \in S$ for $t$ close to $t_{k}$ and $m(\lambda(t), u(t))$ changes across $t_{k}$. If $\lambda(t)$ is injective, by Proposition 2.7 in [1] we have that $\left(\lambda\left(t_{k}\right), u\left(t_{k}\right)\right)$ is a bifurcation point. Then we get the validity of Conjecture 1 as claimed below.

Theorem 2.3. Assume $2 \leq N \leq 7$ and $f$ be as in (1). Then there exists a continuous, piecewise analytic curve $(\lambda(t), u(t))_{t \geq 0}$ in $\mathcal{V}$, starting from $(0,0)$ and so that $\|\hat{u}(t)\|_{\infty} \rightarrow 1$ as $t \rightarrow+\infty$, which has either infinitely many turning points, i.e. points where $(\lambda(t), u(t))$ changes direction (the branch locally "bends back"), or infinitely many bifurcation points.

REMARK 2.1. In [7] the above analysis is performed in the radial setting to obtain a curve $(\lambda(t), u(t))_{t \geq 0}$, as given by Theorem 2.3, composed by radial solutions and so that $m_{r}(\lambda(t), u(t)) \rightarrow+\infty$ as $t \rightarrow+\infty, m_{r}(\lambda, u)$ being the radial Morse index of a solution $(\lambda, u)$. In this way, it can be shown that bifurcation points can't occur and then $(\lambda(t), u(t))_{t \geq 0}$ exhibits infinitely many turning points. Moreover, they can also deal with the case where $N \geq 8$ and $\alpha>\alpha_{N}$.

3. Uniqueness of solutions for small voltage in star-shaped domains. We address the issue of uniqueness of solutions of the singular elliptic problem

$$
\begin{cases}-\Delta u=\frac{\lambda|x|^{\alpha}}{(1-u)^{2}} & \text { in } \Omega \\ 0<u<1 & \text { in } \Omega \\ u=0 & \text { on } \partial \Omega\end{cases}
$$

for $\lambda>0$ small, where $\alpha \geq 0$ and $\Omega$ is a bounded domain in $\mathbb{R}^{N}, N \geq 2$. We shall make crucial use of the following extension of Pohozaev's identity due to Pucci and Serrin [12].

Proposition 3.1. Let $v$ be a solution of the boundary value problem

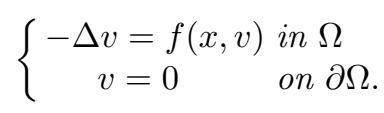


Then for any $a \in \mathbb{R}$ and any $h \in C^{2}\left(\Omega ; \mathbb{R}^{N}\right) \cap C^{1}\left(\bar{\Omega} ; \mathbb{R}^{N}\right)$, the following identity holds

$$
\begin{aligned}
& \int_{\Omega}\left[\operatorname{div}(h) F(x, v)-\operatorname{avf}(x, v)+\left\langle\nabla_{x} F(x, v), h\right\rangle\right] d x \\
= & \int_{\Omega}\left[\left(\frac{1}{2} \operatorname{div}(h)-a\right)|\nabla v|^{2}-\langle D h \nabla v, \nabla v\rangle\right] d x+\frac{1}{2} \int_{\partial \Omega}|\nabla v|^{2}\langle h, \nu\rangle d \sigma,
\end{aligned}
$$

where $F(x, s)=\int_{0}^{s} f(x, t) d t$.

An application of the method in [13] leads to the following result.

TheOREM 3.1. Let $\Omega \subset \mathbb{R}^{N}$ be a star-shaped domain with respect to 0 . If $N \geq 3$, then for $\lambda$ small, the stable solution $u_{\lambda}$ is the unique solution of equation (4).

Proof. Since $u_{\lambda}$ is the minimal solution of (4) for $\lambda \in\left(0, \lambda^{*}\right)$, setting $v=u-u_{\lambda}$ equation (4) rewrites equivalently as

$$
\begin{cases}-\Delta v=\lambda|x|^{\alpha} g_{\lambda}(x, v) & \text { in } \Omega \\ 0 \leq v<1-u_{\lambda} & \text { in } \Omega \\ v=0 & \text { on } \partial \Omega\end{cases}
$$

where

$$
g_{\lambda}(x, s)=\frac{1}{\left(1-u_{\lambda}(x)-s\right)^{2}}-\frac{1}{\left(1-u_{\lambda}(x)\right)^{2}}
$$

It then suffices to prove that the solutions of (6) must be trivial for $\lambda$ small enough. First compute $G_{\lambda}(x, s)$ :

$$
G_{\lambda}(x, s)=\int_{0}^{s} g_{\lambda}(x, t) d t=\frac{1}{1-u_{\lambda}(x)-s}-\frac{1}{1-u_{\lambda}(x)}-\frac{s}{\left(1-u_{\lambda}(x)\right)^{2}} .
$$

Since the validity of the relation

$$
\nabla_{x}\left(|x|^{\alpha} G_{\lambda}(x, s)\right)=\alpha|x|^{\alpha-2} x G_{\lambda}(x, s)+|x|^{\alpha} \nabla_{x} G_{\lambda}(x, s),
$$

for $h(x)=\frac{x}{N}$ and $f(x, v)=|x|^{\alpha} g_{\lambda}(x, v)$ we apply the Pohozaev identity (5) to a solution $v$ of $(6)$ to get

$$
\begin{aligned}
& \lambda \int_{\Omega}|x|^{\alpha}\left[\left(1+\frac{\alpha}{N}\right) G_{\lambda}(x, v(x))-a v(x) g_{\lambda}(x, v(x))+\left\langle\nabla_{x} G_{\lambda}(x, v(x)), \frac{x}{N}\right\rangle\right] d x \\
& =\int_{\Omega}\left[\left(\frac{1}{2}-a\right)|\nabla v|^{2}-\left\langle D\left(\frac{x}{N}\right) \nabla v, \nabla v\right\rangle\right] d x+\frac{1}{2 N} \int_{\partial \Omega}|\nabla v|^{2}\langle x, \nu\rangle d \sigma \\
& \geq\left(\frac{1}{2}-a-\frac{1}{N}\right) \int_{\Omega}|\nabla v|^{2} d x
\end{aligned}
$$

Since easy calculations show that

$$
\frac{G_{\lambda}(x, s)}{g_{\lambda}(x, s)}=\frac{1-u_{\lambda}(x)-s-\frac{\left(1-u_{\lambda}(x)-s\right)^{2}\left(1-u_{\lambda}(x)+s\right)}{\left(1-u_{\lambda}(x)\right)^{2}}}{1-\frac{\left(1-u_{\lambda}(x)-s\right)^{2}}{\left(1-u_{\lambda}(x)\right)^{2}}}
$$


and

$$
\frac{\nabla_{x} G_{\lambda}(x, s)}{g_{\lambda}(x, s)}=\frac{1-\frac{\left(1-u_{\lambda}(x)-s\right)^{2}\left(1-u_{\lambda}(x)+2 s\right)}{\left(1-u_{\lambda}(x)\right)^{3}}}{1-\frac{\left(1-u_{\lambda}(x)-s\right)^{2}}{\left(1-u_{\lambda}(x)\right)^{2}}} \nabla u_{\lambda}(x),
$$

we obtain

$$
\left|\frac{G_{\lambda}(x, s)}{g_{\lambda}(x, s)}\right| \leq C_{0}\left|1-u_{\lambda}(x)-s\right| \quad \text { and } \quad\left|\frac{\nabla_{x} G_{\lambda}(x, s)}{g_{\lambda}(x, s)}-\nabla u_{\lambda}\right| \leq C_{0}\left|1-u_{\lambda}(x)-s\right|^{2}\left|\nabla u_{\lambda}\right|
$$

for some $C_{0}>0$, provided $\lambda$ is away from $\lambda^{*}$. Since $u_{\lambda} \rightarrow 0$ in $C^{1}(\bar{\Omega})$ as $\lambda \rightarrow 0^{+}$, for $a>0$ from (9) we deduce that for any $(x, s)$ satisfying $\left|1-u_{\lambda}(x)-s\right| \leq \delta$

$$
\begin{aligned}
& \left(1+\frac{\alpha}{N}\right) G_{\lambda}(x, s)-\operatorname{asg}_{\lambda}(x, s)+\left\langle\nabla_{x} G_{\lambda}(x, s), \frac{x}{N}\right\rangle \\
& \leq g_{\lambda}(x, s)\left[C_{0}\left(1+\frac{\alpha}{N}\right) \delta-a\left(1-u_{\lambda}(x)-\delta\right)+\left\langle\nabla u_{\lambda}, \frac{x}{N}\right\rangle+\frac{C_{0}}{N} \delta^{2}\left|\nabla u_{\lambda}\right||x|\right] \leq 0,
\end{aligned}
$$

provided $\delta$ and $\lambda$ are sufficiently small (depending on $a$ ). Since $N \geq 3$, we can pick $0<a<\frac{1}{2}-\frac{1}{N}$, and then by (8), (10) get that

$$
\begin{aligned}
& \lambda \int_{\left\{0 \leq v \leq 1-u_{\lambda}-\delta\right\}}|x|^{\alpha}\left[\left(1+\frac{\alpha}{N}\right) G_{\lambda}(x, v(x))-a v(x) g_{\lambda}(x, v(x))+\left\langle\nabla_{x} G_{\lambda}(x, v(x)), \frac{x}{N}\right\rangle\right] d x \\
& \geq\left(\frac{1}{2}-a-\frac{1}{N}\right) \int_{\Omega}|\nabla v|^{2} d x \geq C_{s}\left(\frac{1}{2}-a-\frac{1}{N}\right) \int_{\Omega} v^{2} d x
\end{aligned}
$$

for $\delta$ and $\lambda$ sufficiently small, where $C_{s}$ is the best constant in the Sobolev embedding of $H_{0}^{1}(\Omega)$ into $L^{2}(\Omega)$.

On the other hand, since $G_{\lambda}(x, s), s g_{\lambda}(x, s)$ and $\nabla_{x} G_{\lambda}(x, s)$ are quadratic with respect to $s$ as $s \rightarrow 0$ (uniformly in $\lambda$ away from $\lambda^{*}$ ), there exists a constant $C_{\delta}>0$ such that

$$
\left(1+\frac{\alpha}{N}\right) G_{\lambda}(x, v(x))-\operatorname{avg}_{\lambda}(x, v(x))+\left\langle\nabla_{x} G_{\lambda}(x, v(x)), \frac{x}{N}\right\rangle \leq C_{\delta} v^{2}(x)
$$

for $x \in\left\{0 \leq v \leq 1-u_{\lambda}-\delta\right\}$, uniformly for $\lambda$ away from $\lambda^{*}$. Combining (11) and (12) we get that

$$
C_{s}\left(\frac{1}{2}-a-\frac{1}{N}\right) \int_{\left\{0 \leq v \leq 1-u_{\lambda}-\delta\right\}} v^{2} d x \leq \lambda C_{\delta} \int_{\left\{0 \leq v \leq 1-u_{\lambda}-\delta\right\}}|x|^{\alpha} v^{2} d x .
$$

Therefore, for $\lambda$ sufficiently small we conclude that $v \equiv 0$ in $\left\{0 \leq v \leq 1-u_{\lambda}-\delta\right\}$. This implies that $v \equiv 0$ in $\Omega$ for sufficiently small $\lambda$, and we are done.

We now refine the above argument so as to cover other situations. To this aim, we consider the - potentially empty - set

$$
H(\Omega)=\left\{h \in \mathcal{C}^{1}\left(\bar{\Omega}, \mathbb{R}^{N}\right): \operatorname{div}(h) \equiv 1 \text { and }\langle h, \nu\rangle \geq 0 \text { on } \partial \Omega\right\},
$$

and the corresponding parameter

$$
M(\Omega):=\inf \left\{\sup _{x \in \Omega} \bar{\mu}(h, x): h \in H(\Omega)\right\},
$$


where

$$
\bar{\mu}(h, x)=\frac{1}{2} \sup _{|\xi|=1}\left\langle\left(D h(x)+D h(x)^{T}\right) \xi, \xi\right\rangle .
$$

The following is an extension of Theorem 3.1.

TheOREm 3.2. Let $\Omega$ be a bounded domain in $\mathbb{R}^{N}$ such that $M(\Omega)<\frac{1}{2}$. Then, for $\lambda$ small the minimal solution $u_{\lambda}$ is the unique solution of problem (4), provided either $N \geq 3$ or $\alpha>0$.

Proof. As above, we shall prove that equation (6), with $g_{\lambda}$ as in (7), has only trivial solutions for $\lambda$ small. For a solution $v$ of (6) the Pohozaev identity (5) with $h \in H(\Omega)$ yields

$$
\begin{aligned}
& \lambda \int_{\Omega}|x|^{\alpha}\left[G_{\lambda}(x, v(x))\left(1+\alpha\left\langle\frac{x}{|x|^{2}}, h\right\rangle\right)-a v(x) g_{\lambda}(x, v(x))+\left\langle\nabla_{x} G_{\lambda}(x, v(x)), h\right\rangle\right] d x \\
& =\int_{\Omega}\left[\left(\frac{1}{2}-a\right)|\nabla v|^{2}-\frac{1}{2}\left\langle\left(D h+D h^{T}\right) \nabla v, \nabla v\right\rangle\right] d x+\frac{1}{2} \int_{\partial \Omega}|\nabla v|^{2}\langle h, \nu\rangle d \sigma \\
& \geq \int_{\Omega}\left(\frac{1}{2}-a-\bar{\mu}(h, x)\right)|\nabla v|^{2} d x
\end{aligned}
$$

Fix $0<a<\frac{1}{2}-M(\Omega)$ and choose $h \in H(\Omega)$ such that

$$
\frac{1}{2}-a-\sup _{x \in \Omega} \bar{\mu}(h, x)>0 .
$$

It follows from (9) that for any $(x, s)$ satisfying $\left|1-u_{\lambda}(x)-s\right| \leq \delta|x|$ there holds

$$
\begin{aligned}
& G_{\lambda}(x, s)\left(1+\alpha\left\langle\frac{x}{|x|^{2}}, h\right\rangle\right)-\operatorname{avg}_{\lambda}(x, s)+\left\langle\nabla_{x} G_{\lambda}(x, s), h\right\rangle \\
& \leq g_{\lambda}(x, s)\left[C_{0} \delta|x|+\alpha C_{0} \delta|h|-a\left(1-u_{\lambda}-\delta|x|\right)+\left\langle\nabla u_{\lambda}, h\right\rangle+C_{0} \delta^{2}|x|^{2}\left|\nabla u_{\lambda}\right||h|\right] \leq 0
\end{aligned}
$$

provided $\lambda$ and $\delta$ are sufficiently small. It then follows from (13) and (15) that

$$
\begin{aligned}
& \lambda \int_{\left\{0 \leq v \leq 1-u_{\lambda}-\delta|x|\right\}}|x|^{\alpha}\left[G_{\lambda}(x, v(x))\left(1+\alpha\left\langle\frac{x}{|x|^{2}}, h\right\rangle\right)\right. \\
& \left.\quad-a v(x) g_{\lambda}(x, v(x))+\left\langle\nabla_{x} G_{\lambda}(x, v(x)), h\right\rangle\right] d x \\
& \geq\left(\frac{1}{2}-a-\sup _{x \in \Omega} \bar{\mu}(h, x)\right) \int_{\Omega}|\nabla v|^{2} d x .
\end{aligned}
$$

On the other hand, there exists a constant $C_{\delta}>0$ such that

$$
\begin{aligned}
& G_{\lambda}(x, v(x))\left(1+\alpha\left\langle\frac{x}{|x|^{2}}, h(x)\right\rangle\right)-a v(x) g_{\lambda}(x, v(x))+<\nabla_{x} G_{\lambda}(x, v(x)), h(x)> \\
& =\frac{v^{2}(x)}{\left(1-u_{\lambda}(x)-v(x)\right)\left(1-u_{\lambda}(x)\right)^{2}}\left(1+\alpha\left\langle\frac{x}{|x|^{2}}, h(x)\right\rangle\right)+\frac{\left.a v^{2}(x)\left[v(x)-2+2 u_{\lambda}(x)\right)\right]}{\left(1-u_{\lambda}(x)-v(x)\right)^{2}\left(1-u_{\lambda}(x)\right)^{2}} \\
& +\frac{v^{2}(x)\left(3-3 u_{\lambda}(x)-2 v(x)\right)}{\left(1-u_{\lambda}(x)-v(x)\right)^{2}\left(1-u_{\lambda}(x)\right)^{3}}<\nabla u_{\lambda}(x), h(x)>\leq C_{\delta} \frac{v^{2}(x)}{|x|^{2}}
\end{aligned}
$$


for $x \in\left\{0 \leq v \leq 1-u_{\lambda}-\delta|x|\right\}$, uniformly for $\lambda$ away from $\lambda^{*}$.

If now $N \geq 3$, then Hardy's inequality combined with (15) implies

$$
\frac{(N-2)^{2}}{4}\left(\frac{1}{2}-a-\sup _{x \in \Omega} \bar{\mu}(h, x)\right) \int_{\left\{0 \leq v \leq 1-u_{\lambda}-\delta|x|\right\}} \frac{v^{2}}{|x|^{2}} d x \leq \lambda C_{\delta} \int_{\left\{0 \leq v \leq 1-u_{\lambda}-\delta|x|\right\}} \frac{v^{2}}{|x|^{2}} d x .
$$

On the other hand, when $N=2$ the space $H_{0}^{1}(\Omega)$ embeds continously into $L^{p}(\Omega)$ for every $p>1$, and then, by Hölder inequality, for $\alpha>0$ we get that

$$
\int_{\Omega} \frac{v^{2}}{|x|^{2-\alpha}} d x \leq\left(\int_{\Omega}|x|^{-(2-\alpha) \frac{p}{p-2}} d x\right)^{\frac{p-2}{p}}\left(\int_{\Omega}|v|^{p} d x\right)^{\frac{2}{p}} \leq C_{N, \alpha}^{-1} \int_{\Omega}|\nabla v|^{2} d x
$$

provided $(2-\alpha) \frac{p}{p-2}<2$, which is true for $p$ large depending on $\alpha$ (see [6] for some very general Hardy inequalities). It combines with (15) to yield

$C_{N, \alpha}\left(\frac{1}{2}-a-\sup _{x \in \Omega} \bar{\mu}(h, x)\right) \int_{\left\{0 \leq v \leq 1-u_{\lambda}-\delta|x|\right\}} \frac{v^{2}}{|x|^{2-\alpha}} d x \leq \lambda C_{\delta} \int_{\left\{0 \leq v \leq 1-u_{\lambda}-\delta|x|\right\}} \frac{v^{2}}{|x|^{2-\alpha}} d x$.

In both cases, we can conclude that for $\lambda$ sufficiently small $v \equiv 0$ for $x \in\{0 \leq v \leq$ $\left.1-u_{\lambda}-\delta|x|\right\}$, for some $\delta>0$ small. Since we can assume $\delta$ and $\lambda$ sufficiently small to have

$$
1-u_{\lambda}-\delta|x| \geq \frac{1}{2} \quad \text { in } \quad\left\{x \in \Omega:|x| \geq \frac{1}{2} \operatorname{dist}(0, \partial \Omega)\right\},
$$

we then have

$$
v \equiv 0 \quad \text { in } \quad\left\{x \in \Omega: v(x) \leq \frac{1}{2}\right\} \cap\left\{x \in \Omega:|x| \geq \frac{1}{2} \operatorname{dist}(0, \partial \Omega)\right\} .
$$

Since $v=0$ on $\partial \Omega$ and the domain $\left\{x \in \Omega:|x| \geq \frac{1}{2} \operatorname{dist}(0, \partial \Omega)\right\}$ is connected, the continuity of $v$ gives that

$$
v \equiv 0 \quad \text { in } \quad\left\{x \in \Omega:|x| \geq \frac{1}{2} \operatorname{dist}(0, \partial \Omega)\right\} .
$$

Therefore, the maximum principle for elliptic equations implies $v \equiv 0$ in $\Omega$, which completes the proof of Theorem 3.2.

REMARK 3.1. In [13] examples of dumbell shaped domains $\Omega \subset \mathbb{R}^{N}$ which satisfy condition $M(\Omega)<\frac{1}{2}$ are given for $N \geq 3$. When $N \geq 4$, there even exist topologically nontrivial domains with this property. Let us stress that in both cases $\Omega$ is not starlike, which means that the assumption $M(\Omega)<\frac{1}{2}$ on a domain $\Omega$ is more general than being shar-shaped.

The remaining case $N=2$ and $\alpha=0$, is a bit more delicate. We have the following result.

THEOREM 3.3. If $\Omega$ is either a strictly convex or a symmetric domain in $\mathbb{R}^{2}$, then $(S)_{\lambda, 1}$ has the unique solution $u_{\lambda}$ for small $\lambda$.

Proof. The crucial point here is the following inequality: for every solution $v$ of (6) there holds

$$
\int_{\partial \Omega}|\nabla v|^{2} d \sigma \geq l(\partial \Omega)^{-1}\left(\int_{\Omega}|\Delta v| d x\right)^{2} .
$$


Indeed, we have that

$$
\begin{aligned}
\int_{\partial \Omega}|\nabla v|^{2} d \sigma \geq l(\partial \Omega)^{-1}\left(\int_{\partial \Omega}|\nabla v| d \sigma\right)^{2} & =l(\partial \Omega)^{-1}\left(\int_{\partial \Omega} \partial_{\nu} v d \sigma\right)^{2} \\
& =l(\partial \Omega)^{-1}\left(\int_{\Omega}|\Delta v| d x\right)^{2}
\end{aligned}
$$

where $l(\partial \Omega)$ is the length of $\partial \Omega$. Note that $-\Delta v=\lambda g_{\lambda}(x, v) \geq 0$ for every solution $u_{\lambda}+v$ of $(S)_{\lambda, 1}$, in view of the minimality of $u_{\lambda}$.

By Lemma 4 in [13] for $\lambda$ small there exists $x_{\lambda} \in \Omega$ so that

$$
\left\langle\nabla u_{\lambda}(x), x-x_{\lambda}\right\rangle \leq 0 \quad \forall x \in \Omega .
$$

In particular, for $\lambda$ small $x_{\lambda}$ lies in a compact subset of $\Omega$ and, when $\Omega$ is symmetric, coincides exactly with the center of symmetries. In both situations, then we have that there exists $c_{0}>0$ so that

$$
\left\langle x-x_{\lambda}, \nu(x)\right\rangle \geq c_{0} \quad \forall x \in \partial \Omega .
$$

We use now the Pohozaev identity (5) with $a=0$ and $h(x)=\frac{x-x_{\lambda}}{2}$. For every solution $v$ of (6) it yields

$$
\begin{aligned}
& \lambda \int_{\Omega}\left[G_{\lambda}(x, v(x))+\left\langle\nabla_{x} G_{\lambda}(x, v(x)), \frac{x-x_{\lambda}}{2}\right\rangle\right] d x \\
= & \frac{1}{4} \int_{\partial \Omega}|\nabla v|^{2}\left\langle x-x_{\lambda}, \nu\right\rangle d \sigma \geq \frac{c_{0}}{4}\left(\int_{\Omega}|\Delta v| d x\right)^{2} .
\end{aligned}
$$

Since

$$
\nabla_{x} G_{\lambda}(x, s)=\left(1-u_{\lambda}(x)-s\right)^{-2}\left[1-\frac{\left(1-u_{\lambda}(x)-s\right)^{2}\left(1-u_{\lambda}(x)+2 s\right)}{\left(1-u_{\lambda}(x)\right)^{3}}\right] \nabla u_{\lambda}(x),
$$

by (16) we easily see that

$$
\left\langle\nabla_{x} G_{\lambda}(x, s), x-x_{\lambda}\right\rangle \leq 0
$$

for $\lambda$ and $\delta$ small, provided $(x, s)$ satisfies $\left|1-u_{\lambda}(x)-s\right| \leq \delta$. Since $G_{\lambda}(x, s)$, $\nabla_{x} G_{\lambda}(x, s)$ are quadratic with respect to $s$ as $s \rightarrow 0$ (uniformly in $\lambda$ small), there exists a constant $C_{\delta}>0$ such that

$$
G_{\lambda}(x, v(x)) \leq C_{\delta} v^{2}(x), \quad\left\langle\nabla_{x} G_{\lambda}(x, v(x)), \frac{x-x_{\lambda}}{2}\right\rangle \leq C_{\delta} v^{2}(x)
$$

for $x \in\left\{0 \leq v \leq 1-u_{\lambda}-\delta\right\}$, uniformly for $\lambda$ small.

Since on two-dimensional domains

$$
\left(\int_{\Omega}|v|^{p} d x\right)^{\frac{1}{p}} \leq C_{p} \int_{\Omega}|\Delta v| d x
$$

for every $p \geq 1$ and $v \in W^{2,1}(\Omega)$ so that $v=0$ on $\partial \Omega$, we get that

$$
\lambda \int_{\Omega}\left\langle\nabla_{x} G_{\lambda}(x, v(x)), \frac{x-x_{\lambda}}{2}\right\rangle d x \leq \lambda C_{\delta} \int_{\Omega} v^{2} d x \leq \lambda C_{\delta} C_{2}^{2}\left(\int_{\Omega}|\Delta v| d x\right)^{2} .
$$


As far as the term with $G_{\lambda}(x, v(x))$, fix $b \in(0,1)$ and split $\Omega$ as the disjoint union of $\Omega_{1}=\{v \leq b\}$ and $\Omega_{2}=\{v>b\}$. On $\Omega_{1}$ we have that

$$
\lambda \int_{\Omega_{1}} G_{\lambda}(x, v(x)) d x \leq \lambda C_{\delta} \int_{\Omega} v^{2} d x \leq \lambda C_{\delta} C_{2}^{2}\left(\int_{\Omega}|\Delta v| d x\right)^{2}
$$

provided $\lambda$ and $\delta$ are small to satisfy $b \leq 1-u_{\lambda}-\delta$ in $\Omega_{1}$.

Since for $\lambda$ small

$$
\frac{G_{\lambda}(x, s)^{2}}{g_{\lambda}(x, s)} \leq C \quad \forall b \leq s \leq 1,
$$

we have that

$$
\begin{aligned}
\lambda \int_{\Omega_{2}} G_{\lambda}(x, v(x)) d x & \leq \lambda D_{1} \int_{\Omega}|v(x)|^{\frac{3}{2}} g_{\lambda}^{\frac{1}{2}}(x, v(x)) d x \\
& \leq \lambda D_{2}\left(\int_{\Omega}|v|^{3} d x\right)^{\frac{1}{2}}\left(\int_{\Omega} g_{\lambda}(x, v(x)) d x\right)^{\frac{1}{2}} \\
& \leq \lambda^{\frac{1}{2}} D_{3}\left(\int_{\Omega}|\Delta v| d x\right)^{2}
\end{aligned}
$$

for some positive constants $D_{1}, D_{2}$ and $D_{3}$. So we get that

$$
\lambda \int_{\Omega} G_{\lambda}(x, v(x)) d x \leq\left(\lambda C_{\delta} C_{2}^{2}+\lambda^{\frac{1}{2}} D_{3}\right)\left(\int_{\Omega}|\Delta v| d x\right)^{2} .
$$

Inserting (18)-(19) into (17) finally we get that

$$
\left(2 \lambda C_{\delta} C_{2}^{2}+\lambda^{\frac{1}{2}} D_{3}-\frac{c_{0}}{4}\right)\left(\int_{\Omega}|\Delta v| d x\right)^{2} \geq 0
$$

and then $v \equiv 0$ for $\lambda$ small.

\section{REFERENCES}

[1] B. Buffoni, E.N. Dancer and J.F. Toland, The sub-harmonic bifurcation of Stokes waves, Arch. Ration. Mech. Anal., 152:3 (2000), pp. 241-271.

[2] E.N. DANCer, Infinitely many turning points for some supercritical problems, Ann. Mat. Pura Appl., 178:4 (2000), pp. 225-233.

[3] P. Esposito, Compactness of a nonlinear eigenvalue problem with a singular nonlinearity, Commun. Contemp. Math., 10:1 (2008), pp. 17-45.

[4] P. Esposito, N. Ghoussoub And Y. Guo, Compactness along the branch of semi-stable and unstable solutions for an elliptic problem with a singular nonlinearity, Comm. Pure Appl. Math., 60:12 (2007), pp. 1731-1768.

[5] N. Ghoussoub And Y. Guo, On the partial differential equations of electrostatic MEMS devices: stationary case, SIAM J. Math. Anal., 38:5 (2006/2007), pp. 1423-1449.

[6] N. Ghoussoub and A. Moradifam, On the best possible remaining term in the improved Hardy inequality, Proc. Nat. Acad. Sci., 105:37 (2008), pp. 13746-13751.

[7] Z. Guo And J. WeI, Infinitely many turning points for an elliptic problem with a singular nonlinearity, J. Lond. Math. Soc. (2), to appear.

[8] Z. Guo And J. Wei, Asymptotic behavior of touch-down solutions and global bifurcations for an elliptic problem with a singular nonlinearity, Commun. Pure Appl. Anal., 7:4 (2008), pp. $765-786$.

[9] D.D. Joseph And T.S. Lundgren, Quasilinear Dirichlet problems driven by positive sources, Arch. Rational Mech. Anal., 49 (1972/73), pp. 241-269. 
[10] J.A. Pelesko, Mathematical modeling of electrostatic MEMS with tailored dielectric properties, SIAM J. Appl. Math., 62:3 (2001/2002), pp. 888-908.

[11] J.A. Pelesko and D.H. Bernstein, Modeling MEMS and NEMS. Chapman \& Hall/CRC, Boca Raton, FL, 2003.

[12] P. Pucci and J. Serrin, A general variational identity, Indiana Univ. Math. J., 35:3 (1986), pp. 681-703.

[13] R. SCHAAF, Uniqueness for semilinear elliptic problems: supercritical growth and domain geometry, Adv. Differential Equations, 5:10-12 (2000), pp. 1201-1220. 
P. ESPOSITO AND N. GHOUSSOUB 